\title{
Restriction of task processing time affects cortical activity during processing of a cognitive task: an event-related slow cortical potential study
}

\author{
Claus Lamm*, Herbert Bauer, Oliver Vitouch, Susanne Durec, Roswitha Gronister, \\ Reinhard Gstättner
}

Brain Research Laboratory, Department of Psychology, University of Vienna, Liebiggasse 5, A-1010 Vienna, Austria

Accepted 25 July 2000

\begin{abstract}
As is known from psychometrics, restriction of task processing time by the instruction to respond as quickly and accurately as possible leads to task-unspecific cognitive processing. Since this task processing mode is used in most functional neuroimaging studies of human cognition, this may evoke cortical activity that is functionally not essential for the particular task under investigation. Using topographic recordings of event-related slow cortical potentials, two experiments have been performed to investigate whether cortical activity during processing of a visuo-spatial imagery task is substantially influenced by the time provided to process the task. Furthermore, it was investigated whether this effect is additionally modulated by a subject's task-specific ability. The instruction to respond as quickly and accurately as possible led to increased negative slow cortical potential amplitudes over parietal and frontal regions and significantly interacted with task-specific ability. While cortical activity recorded over parietal and frontal regions was different between subjects with low and high spatial ability when processing time was unrestricted, no such differences were found between ability groups when subjects were instructed to answer both quickly and accurately. These results suggest that restricting processing time has considerable effects on the amount and the pattern of brain activity during cognitive processing and should be taken into account more explicitly in the experimental design and interpretation of neuroimaging studies of cognition. (C) 2001 Elsevier Science B.V. All rights reserved.
\end{abstract}

Theme: Neural basis of behavior

Topic: Cognition

Keywords: Experimental design; Task processing; Individual differences; Slow cortical potentials; Functional neuroimaging; Visuo-spatial imagery

\section{Introduction}

Different cognitive processes are evoked in subjects solving cognitive tasks when speed and accuracy are required rather than accuracy alone. While the so-called 'power' component of cognitive task processing is assigned to task-specific processing, the 'speed' component is related to more general cognitive processes, such as directed attention, allocation of processing resources, or effort [14,26,34]. The resulting speed-accuracy trade-off [11] might additionally reflect changes in the cognitive

\footnotetext{
*Corresponding author. Tel.: +43-1-4277-47829; fax: +43-1-427747859.

E-mail address: claus.lamm@univie.ac.at (C. Lamm).
}

strategy to process a task. These psychometric findings are usually not considered in the design of functional neuroimaging studies of cognition where it is common practice to restrict task processing time and to instruct subjects to respond as quickly and accurately as possible whenever a task is presented. Since this processing mode might lead to neural activity not directly attributable to the task investigated, it might confound the functional interpretation and generalizability of findings. The effects of time pressure on event-related potentials have been investigated in several studies $[10,16]$. These studies used simple reaction or choice reaction paradigms, and considerable time pressure was induced. The latter also applies to a recent study investigating modulations of several EEG frequency bands by time pressure during execution of a visuomotor task 
[31]. However, whether the rather moderate time pressure induced by the instruction to respond as quickly as possible affects cortical activity during processing of a task requiring higher-order cognitive functions has not yet been investigated systematically. Therefore, we conducted two consecutive experiments in which event-related slow cortical potentials (SCPs) were recorded while subjects were solving a visuo-spatial imagery task either with or without restrictions of task processing time. Task-specific ability of subjects was additionally varied in order to investigate whether the previously observed modulation of cortical activity by ability differences $[18,22,35]$ interacts with the effects of task processing mode.

\section{Methods}

\subsection{Subjects and experimental setting}

Only right-handed [1] healthy volunteers (age range 18-34 years) participated in the two experiments. Subjects were selected out of a large pre-testing sample in which about 600 subjects had been tested for their visuo-spatial imagery ability using a standardized three-dimensional cube comparison test (3DC) [12]. Only subjects whose test performance fell either into the first (poor performers) or into the fourth quartile (good performers) of the age- and sex-specific calibration sample of the 3DC were investigated. Twenty-two (12 males) subjects with high and 16 (8 males) subjects with low test scores participated in experiment I (unrestricted processing time), and an independent sample of 29 (16 males) good and 27 (14 males) poor performers was investigated in experiment II (restricted processing time). Pre-testing also was used to yield comparable levels of experience between subjects in processing 3DC-tasks since they were also used during EEG recording. Each task involved two blue-coloured cubes with white patterns (triangles, squares, crosses etc.) on their faces (see Fig. 1) simultaneously displayed in the middle of a computer screen which was located approximately 70 $\mathrm{cm}$ in front of the subjects. The subjects had to determine whether or not the cubes could be identical. This required the mental rotation/transformation of one of the cubes and a matching to the other cube. Answers were given by
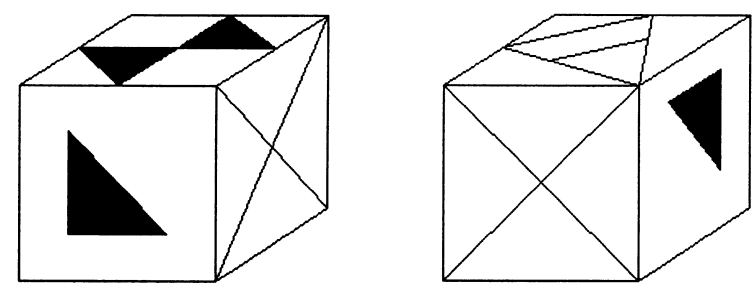

Fig. 1. Black/white sample of a cube comparison task used in the experiments. Correct answer: 'yes - the two cubes could be identical'. In the experiments, cubes were blue with white patterns and edges and presented on black background. pressing one of two response buttons (answers yes/no) mounted on a response board using the dominant right hand. The decision to use 3DC-tasks in the investigation of visuo-spatial imagery was based on several favourable properties of the 3DC task material. Psychometric properties such as reliability and validity are known and high and have been determined using large calibration samples. This is in contrast to most task material used in cognitive neuroimaging where face validity sometimes is the only known psychometric property. 3DC-tasks also allow the assessment of visuo-spatial imagery with high intra- and interindividual homogeneity since they fulfill the criteria of the Rasch model [27]. Tests which conform to this model are unidimensional, which means that identical cognitive processes are involved in all tasks and in different groups of subjects. This is contrary to, e.g., 2-dimensional letter or object comparison tests in which some tasks do not invoke mental rotation of stimuli $[8,9]$, or in which some subjects might dominantly use verbalizing instead of visuo-spatial strategies. Different task processing instructions were used in the two experiments. In experiment I the subjects were informed that there was no time limit for solving each task and hence task processing time would not be restricted (power condition). In experiment II, the subjects were instructed to answer both quickly and accurately since task processing time was restricted (speed condition). However, in order to induce only moderate time pressure and to avoid demotivating or frustrating participants, task presentation time was chosen to be only approximately $10 \%$ shorter than the median processing times of the respective group under power conditions (which corresponded to $14 \mathrm{~s}$ for the poor group and $11 \mathrm{~s}$ for the good group).

\subsection{EEG recording}

EEG was recorded via $22 \mathrm{Ag} / \mathrm{AgCl}$ electrodes attached to small collodion-fixed plastic adapters evenly distributed over the scalp. With the aid of an application cap a $2+4 \times 5$ equidistant matrix montage (locations: $\mathrm{Fp}^{\prime}, \mathrm{Fp} 2^{\prime} / \mathrm{F}^{\prime}$, F3', Fz,F4', F8'/T3', C3', Cz, C4', T4' /T5', P3' $^{\prime}, \mathrm{Pz}, \mathrm{P}^{\prime}$ ', $\mathrm{T}^{\prime} / \mathrm{O}^{\prime}, \mathrm{O}^{\prime}, \mathrm{Oz}, \mathrm{O} 4^{\prime}, \mathrm{O} 8^{\prime}$, all referenced to linked mastoids; due to the equidistance constraint electrodes marked with an apostrophe only approximated the positions specified in the international 10-20 system) was achieved. To minimize skin potential artifacts and to keep electrode impedance reliably $\leq 1 \mathrm{k} \Omega$ skin scratching was used. Electrodes and adapters were filled with degassed electrode gel and connected to a high input impedance $(\geq 100 \mathrm{G} \Omega)$ DC amplifier with high baseline stability. Vertical (electrodes above and below right eye) and horizontal (electrodes on outer canthi) electrooculograms (EOG) were recorded bipolarly in order to eliminate eye movement artifacts offline using a linear regression algorithm (see [35] for a detailed description). Recordings were sampled at $4 \mathrm{kHz}$ (down-sampled to $250 \mathrm{~Hz}$ for digital storage) with the frequency range set from DC to $100 \mathrm{~Hz}$ 
(notch filter at $50 \mathrm{~Hz}$ ). Variable data epochs covering a $2 \mathrm{~s}$ pre-presentation baseline and the entire processing period were stored to disk trial by trial.

\subsection{Data analysis and dependent variables}

Artifact rejection was performed off-line by visual inspection of all trials, with subjects having processed 68 trials in the mean. Trials without responses within the required time limit and with incorrect responses, respectively, were excluded from the analyses. After making sure that there were no systematic or relevant differences in the baseline interval, stimulus onset linked averages of all artifact-free and correctly answered trials were computed with a $200 \mathrm{~ms}$ pre-stimulus baseline for each subject. Averages and their current source density (CSD) transforms were mapped by means of spherical-spline interpolation [25] as a visualization aid for topographic interpretation. CSD (surface Laplacian) transformation was used since it is known to yield reference-free maps and to attenuate low spatial frequencies ('smearing') introduced into the scalp potential distribution due to volume conduction, thus providing a more precise estimate of the epicortical surface potential distribution [23,24]. Since response times were variable within and across subjects in both experiments, an analysis epoch of a length of $5 \mathrm{~s}$ was selected based on the median reaction times. This epoch length was chosen since it was long enough to reliably induce cognition-related SCPs, and since only a few trials had to be rejected due to response times shorter than $5 \mathrm{~s}$. In order to exclude activity from movement preparation and execution related to the button press, only trials with response times $\geq 5500 \mathrm{~ms}$ were included in the averages. For statistical analysis, mean SCP amplitudes were calculated at various latencies (200 ms epochs centred around $1.9,2.4,2.9,3.4,3.9,4.4$ and $4.9 \mathrm{~s}$ after stimulus onset). Greenhouse-Geisser [13] adjusted repeated-measures analyses of variance (ANOVAs) with the between-subject factors EXPERIMENT (power vs. speed) and GROUP (good vs. poor), and the within-subject factor electrode LOCATION (22) were computed with both SCP raw and $z$-normalized amplitude values $[15,20]$. Significant $(P \leq$ 0.05 ) results of ANOVAs were evaluated using hypothesisdriven linear contrasts calculated with specific error variances [3].

\section{Results}

\subsection{Behavioral data}

Table 1 summarizes response times and the percentage of tasks correctly answered. Both were considerably lower when subjects were instructed to respond both quickly and accurately. This reduction was similar for both groups, with good performers answering considerably more tasks
Table 1

Behavioral data of the two experiments

\begin{tabular}{lllrlc}
\hline Experiment $^{\mathrm{a}}$ & Group & $N$ & \multicolumn{1}{c}{ Score } & $\%$ & $\mathrm{RT}_{\text {Median }}$ \\
\hline I & Good & 22 & $16.2 \pm 0.9$ & 91.8 & 12.7 \\
I & Poor & 16 & $5.1 \pm 2.1$ & 72.6 & 14.8 \\
II & Good & 29 & $16.1 \pm 0.9$ & 84.8 & 6.9 \\
II & Poor & 27 & $4.1 \pm 2.4$ & 68.9 & 8.6 \\
\hline
\end{tabular}

${ }^{a}$ Experiment, I/II $=$ unrestricted $/$ restricted task processing time; Group, Good/Poor=good/poor test performers; $N$, sample size; Score, test score in the three dimensional cube comparison test $(k=17$ items; Mean \pm S.D., [12]); \%, percentage of correctly answered tasks; $\mathrm{RT}_{\text {Median }}$, median item processing time (s).

correctly also when processing time was restricted. In experiment II, only about $4 \%$ of trials had to be excluded from the analyses because no response was given within the required time limit. All of the subjects completed the experiments, and none of them reported having been overcharged by the more demanding task processing instruction or the restriction in processing time, respectively.

\subsection{Event-related slow cortical potentials}

Grand mean waveforms for parietal and fronto-medial electrodes are plotted in Fig. 2, and grand mean potential and CSD maps at four latencies are displayed in Fig. 3. In both experiments and both groups, stimulus presentation was followed by a small negative phasic deflection peaking at around $140 \mathrm{~ms}$ and a positive phasic deflection peaking around $350-400 \mathrm{~ms}$. Around $500-700 \mathrm{~ms}$ after stimulus presentation, negative-going SCPs could be observed. SCPs were most pronounced over parieto-occipital regions where they showed gradually increasing amplitudes in the course of task processing. Additional negative-going SCPs with a similar onset time were also observed over central and frontal brain areas (see Fig. 2). While experiment I revealed topographies that clearly differed between the two groups (cf. [18]), the SCP pattern of good and poor performers was basically identical in experiment II. This obvious difference between the two processing modes and their interaction with task-specific ability was confirmed by the statistical analyses (see Table 2). The interaction EXPERIMENT $\times$ LOCATION was significant for all seven latencies, and starting at $3.4 \mathrm{~s}$ (with a tendency towards significance at $2.9 \mathrm{~s}$ ), the interactions GROUP $\times$ LOCATION and EXPERIMENT $\times$ GROUP $\times$ LOCATION were also significant. Based on earlier findings $[18,35]$ and due to the fact that the largest negative difference between experiments was found over the parietal and fronto-medial cortex (see Fig. 3), SCPs of these regions were further evaluated using linear contrasts.

At the $4.9 \mathrm{~s}$ latency, the between-experiment contrasts calculated for the good group revealed significantly higher parietal and fronto-medial activity under the speed than under the power condition (parietal $=\mathrm{P}^{\prime} \mathrm{PzP}^{\prime}: F_{1,90}=$ 


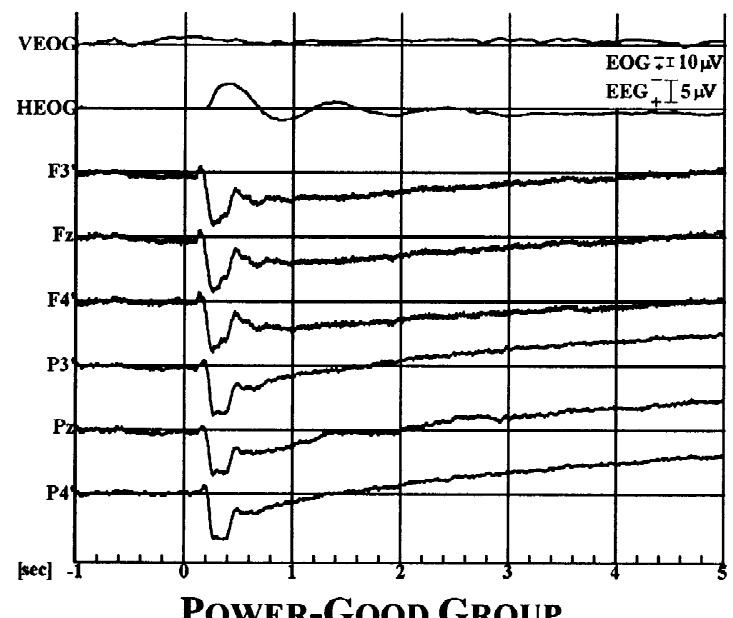

POWER-GoOd GrouP

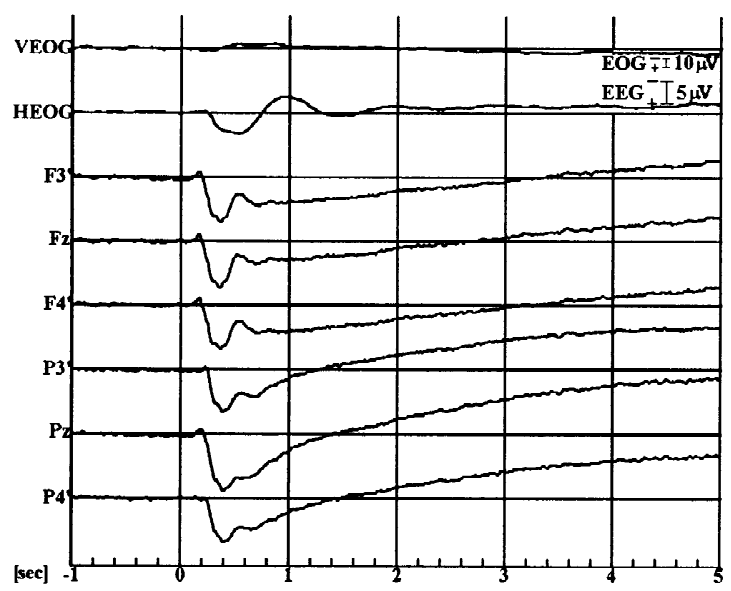

SPEED-GOOD GROUP

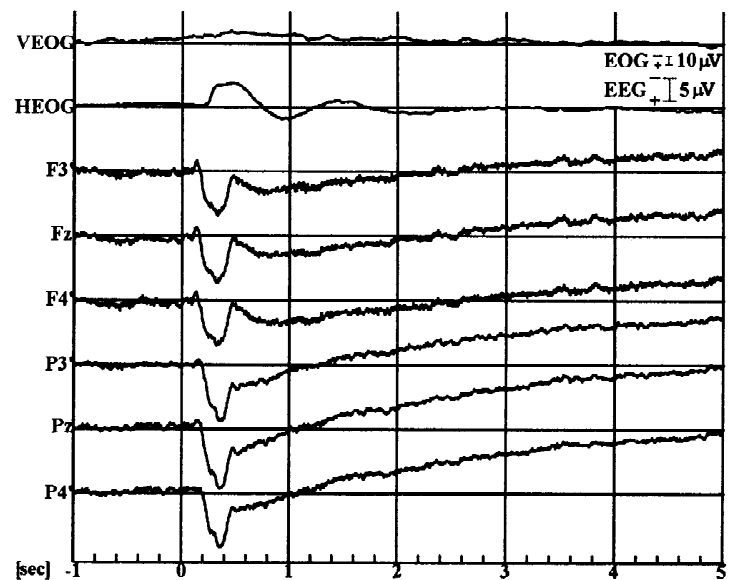

POWER-POOR GrouP

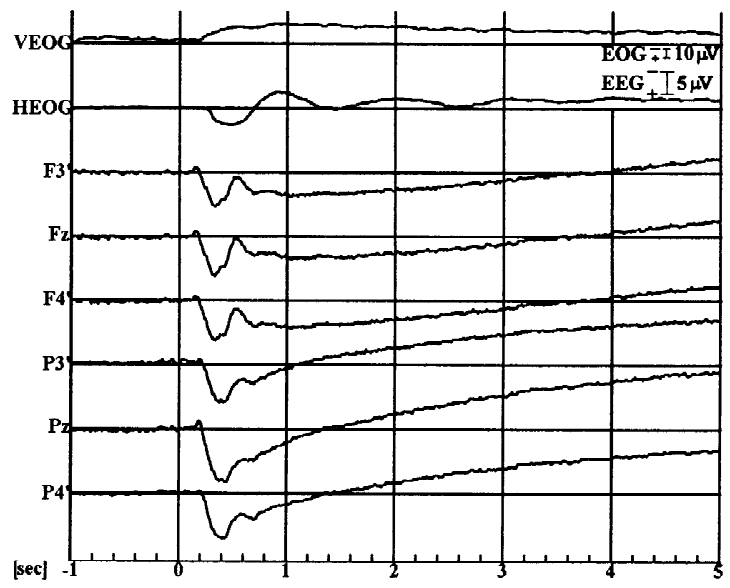

SPEED-POOR GROUP

Fig. 2. Event-related SCP waveforms at parietal and fronto-medial leads of good and poor performers processing a visuo-spatial imagery task with (bottom) and without (top) the instruction to respond as quickly and accurately as possible. Stimulus presentation at $0 \mathrm{~s}$. Note the clear difference in SCP amplitude and time-course between ability groups for parietal as well as frontal recordings when processing time was unrestricted and the almost identical waveforms when processing time was restricted.

4.33, $P=0.04$, mean difference $\Delta=-3.79 \mu \mathrm{V}$; frontomedial $\left.=\mathrm{F}^{\prime}{ }^{\prime} \mathrm{FzF} 4^{\prime}: F_{1,90}=9.02, P=0.003, \Delta=-5.48 \mu \mathrm{V}\right)$. At the $1.9 \mathrm{~s}$ latency, only the fronto-medial contrast was significant $\left(F_{1,90}=4.06, \quad P=0.047, \Delta=-3.00 \mu \mathrm{V}\right)$. No significant results were obtained when the same contrasts were calculated for the poor group. Also, no significant between-group contrasts were observed in experiment II, which contrasted with the significantly higher parietal and fronto-medial activity of poor performers under power conditions (value at 1.9 s: fronto-medial: $F_{1,34}=7.68, P=$ 0.007, $\Delta=-4.80 \mu \mathrm{V}$; parietal: $F_{1,34}=4.38, P=0.039, \Delta=$ $-3.79 \mu \mathrm{V} ;$ value at $4.9 \mathrm{~s}$ : fronto-medial: $F_{1,34}=14.78$, $P<0.001, \Delta=-7.68 \mu \mathrm{V}$; parietal: $F_{1,34}=11.25, P=0.002$, $\Delta=-7.15 \mu \mathrm{V})$. Fig. 4 demonstrates these differences between experiments by showing the SCP means and 95\% confidence intervals of electrodes $\mathrm{Fz}$ and $\mathrm{Pz}$ at the latency of $5 \mathrm{~s}$.

Since it might be hypothesized that the same cognitive processes were evoked in both experiments but at different latencies, we compared the topographies at 4.4, 3.9, 3.4 and $2.9 \mathrm{~s}$ under the speed with those at $4.9 \mathrm{~s}$ under the power condition. The interactions EXPERIMENT $\times$ LOCATION, GROUP $\times$ LOCATION and EXPERIMENT $\times$ GROUP $\times$ LOCATION were significant for all analyses. It is therefore unlikely that the differences between the speed and power condition arose solely from a comparison of differently timed stages of cognitive processing.

\section{Discussion}

Presenting tasks with the instruction to answer as quickly and accurately as possible is the preferred presentation mode in most functional neuroimaging studies. This is at variance with psychometric findings showing that restricting processing time may lead to additional cognitive processing not specifically attributable to the task 


\section{POWER CONDITION}

\section{GOOD}

\section{POOR}

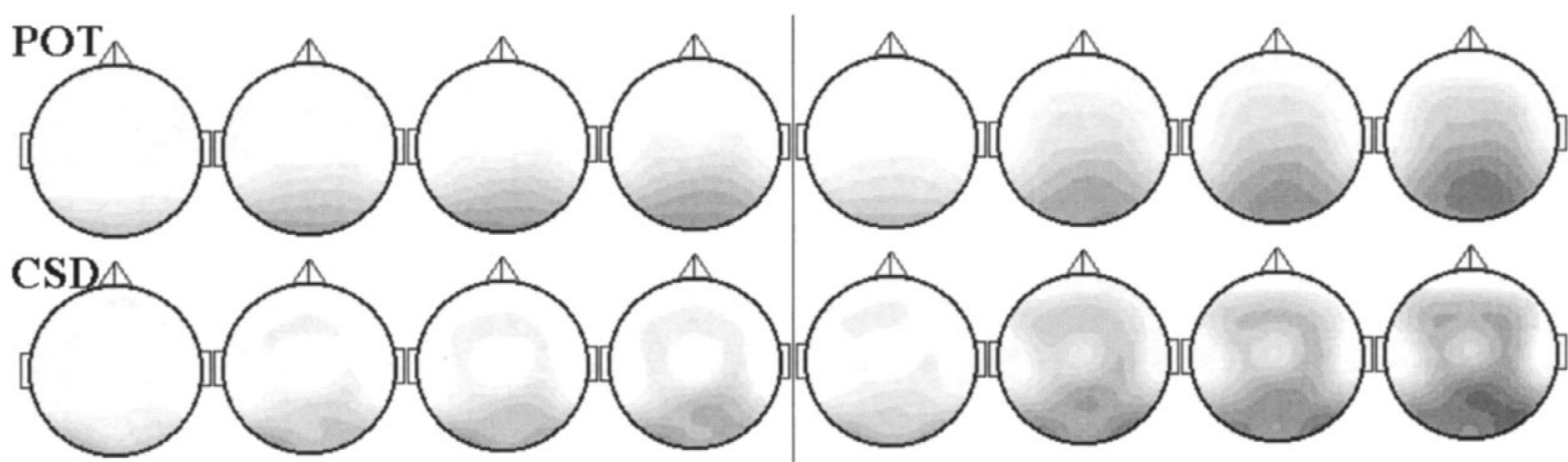

\section{SPEED CONDITION}

GOOD

POOR

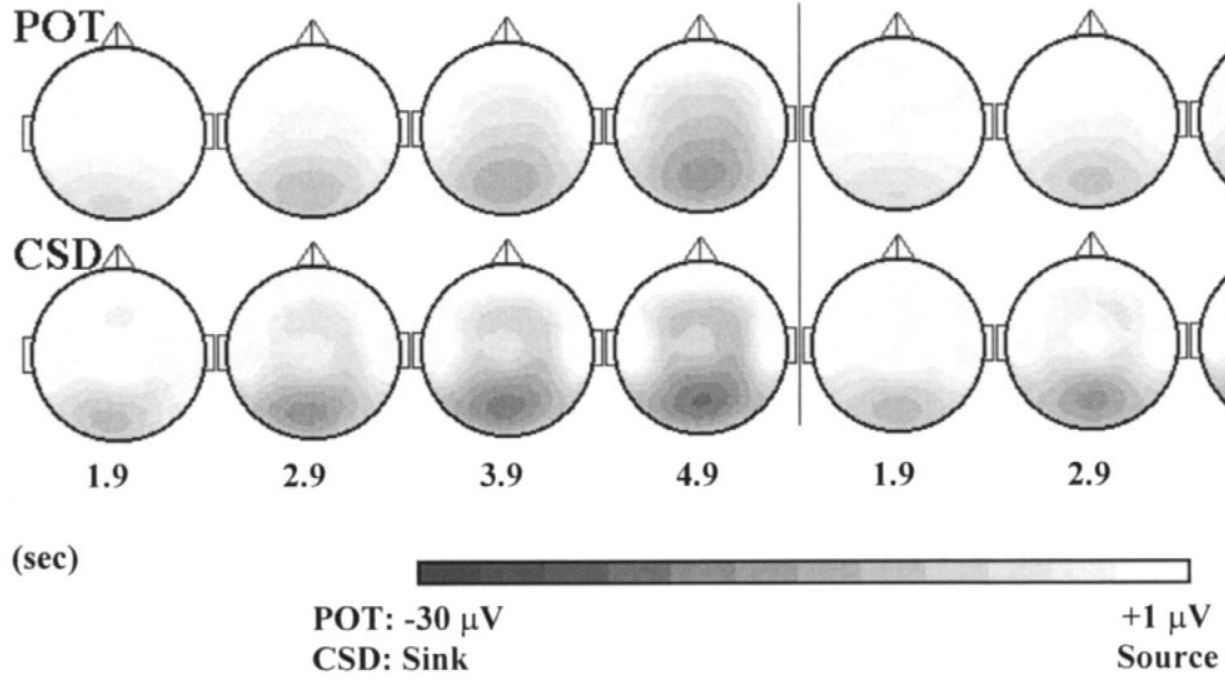

Fig. 3. Slow cortical potential (SCP) topographies of good and poor performers processing a visuo-spatial imagery task with (bottom) and without (top) the instruction to respond as quickly and accurately as possible. Shown are grand mean maps at 1.9, 2.9, 3.9 and 4.9 s post-stimulus. Only negative SCPs are displayed. Upper rows, potential maps (POT); lower rows, current source density maps (CSD). Note that the topographies of the two performance groups were almost identical when processing time was restricted and clearly different when subjects had ample time to solve the tasks.

Table 2

Results of the repeated measures ANOVAs (SCP raw and $z$-normalized amplitude values) at seven latencies (200 ms epochs centred around 1.9, 2.4, 2.9, $3.4,3.9,4.4$ and 4.9 s post-stimulus $^{\mathrm{a}}$

\begin{tabular}{|c|c|c|c|c|c|c|c|c|c|c|c|c|c|c|}
\hline & \multicolumn{2}{|c|}{$\begin{array}{l}1.9 \\
\epsilon_{\mathrm{GG}}=0.18\end{array}$} & \multicolumn{2}{|c|}{$\begin{array}{l}2.4 \\
\epsilon_{\mathrm{GG}}=0.19\end{array}$} & \multicolumn{2}{|c|}{$\begin{array}{l}2.9 \\
\epsilon_{\mathrm{GG}}=0.21\end{array}$} & \multicolumn{2}{|c|}{$\begin{array}{l}3.4 \\
\epsilon_{\mathrm{GG}}=0.23\end{array}$} & \multicolumn{2}{|c|}{$\begin{array}{l}3.9 \\
\epsilon_{\mathrm{GG}}=0.23\end{array}$} & \multicolumn{2}{|c|}{$\begin{array}{l}4.4 \\
\epsilon_{\mathrm{GG}}=0.24\end{array}$} & \multicolumn{2}{|c|}{$\begin{array}{l}4.9 \\
\epsilon_{\mathrm{GG}}=0.26\end{array}$} \\
\hline & $F$ & $P_{\mathrm{GG}}$ & $F$ & $P_{\mathrm{GG}}$ & $F$ & $P_{\mathrm{GG}}$ & $F$ & $P_{\mathrm{GG}}$ & $F$ & $P_{\mathrm{GG}}$ & $F$ & $P_{\mathrm{GG}}$ & $F$ & $P_{\mathrm{GG}}$ \\
\hline \multicolumn{15}{|c|}{ Raw amplitude values } \\
\hline $\mathrm{E} \times \mathrm{L}$ & 2.49 & 0.046 & 3.41 & 0.009 & 3.87 & 0.033 & 5.24 & $<0.001$ & 6.00 & $<0.001$ & 6.31 & $<0.001$ & 9.47 & $<0.001$ \\
\hline $\mathrm{G} \times \mathrm{L}$ & 1.34 & 0.255 & 1.57 & 0.180 & 2.07 & 0.077 & 2.24 & 0.048 & 2.38 & 0.041 & 3.12 & 0.008 & 2.42 & 0.031 \\
\hline $\mathrm{E} \times \mathrm{G} \times \mathrm{L}$ & 1.56 & 0.187 & 2.02 & 0.090 & 2.08 & 0.075 & 2.29 & 0.045 & 2.53 & 0.031 & 2.89 & 0.031 & 2.36 & 0.035 \\
\hline \multicolumn{15}{|c|}{$z$-normalized amplitude values } \\
\hline $\mathrm{E} \times \mathrm{L}$ & 2.36 & 0.039 & 2.25 & 0.042 & 3.20 & 0.004 & 2.89 & 0.008 & 4.59 & $<0.001$ & 5.00 & $<0.001$ & 5.69 & $<0.001$ \\
\hline $\mathrm{G} \times \mathrm{L}$ & 1.89 & 0.094 & 1.58 & 0.157 & 1.98 & 0.063 & 2.20 & 0.039 & 2.17 & 0.034 & 1.98 & 0.066 & 2.29 & 0.029 \\
\hline $\mathrm{E} \times \mathrm{G} \times \mathrm{L}$ & 0.86 & 0.511 & 1.33 & 0.247 & 1.78 & 0.096 & 2.13 & 0.046 & 2.27 & 0.043 & 2.55 & 0.019 & 3.21 & 0.003 \\
\hline
\end{tabular}

Raw amplitude values

${ }^{a}$ E, experiment (unrestricted/restricted task processing time); G, group (good/poor); L, location (22 electrodes); $\epsilon_{\mathrm{GG}}$, sphericity parameter (calculated according to [13]); $P_{\mathrm{GG}}$, adjusted $P$-value. Degrees of freedom (d.f.) of all $F$-values: $\mathrm{df}_{1}($ effect $)=21, \mathrm{df}_{2}($ error $)=1,890$. 


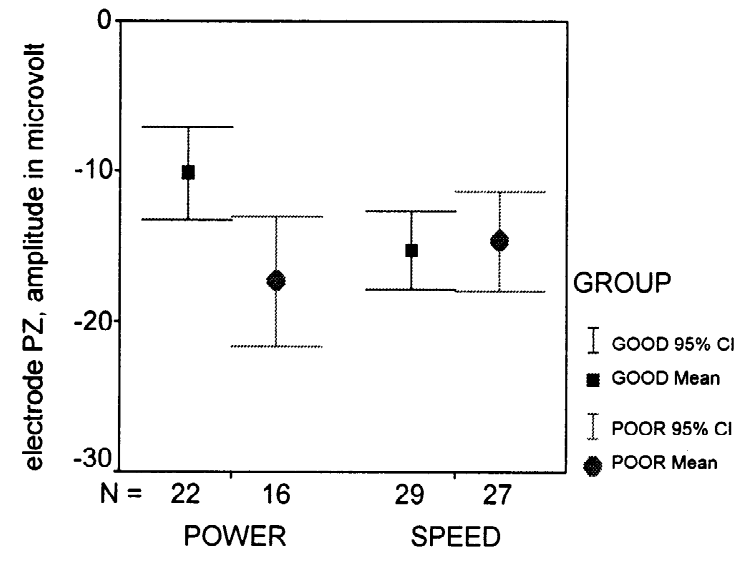

STUDY

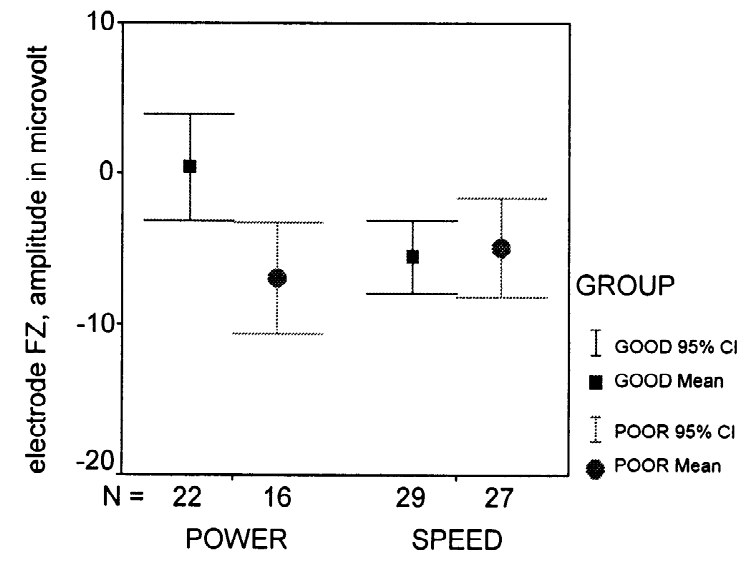

STUDY

Fig. 4. Mean amplitudes $\pm 95 \%$ confidence interval (CI) at the latency value of $5 \mathrm{~s}$ of electrodes $\mathrm{Pz}$ and Fz.

under investigation [14,26,34]. The instruction to respond as quickly and accurately as possible led to a considerable reduction in both response times and the percentage of tasks correctly answered. This is consistent with the wellknown finding of a speed-accuracy trade-off under time pressure [11]; however, the time pressure induced in experiment II was comparably moderate, which might be inferred from the feedback given by the subjects as well as from the rather low rate of missing task completions. The results of our study demonstrate considerable effects of task processing mode on cortical activity, with the instruction to respond both as quickly and accurately as possible evoking additional cortical activity. This was reflected in the significant EXPERIMENT $\times$ LOCATION interactions across the whole analysis period with both raw and $z$ normalized amplitude ANOVAs. While the raw amplitude ANOVAs indicate that the amount of brain activity was affected by the task processing mode, the significant $z$ normalized ANOVAs suggest that also the spatial pattern of cortical activity was substantially influenced - with additional brain areas becoming active when tasks had to be answered within a limited time [15,20]. Since differences in task-specific cognitive ability were also considered in the experiments, the modulation of cortical activity by this variable could be assessed in addition. The threeway ANOVA interactions clearly indicate that it was mainly the cortical activity of good test performers which was affected by the different task processing modes, since their topographies showed increased parietal and frontal activity when processing time was restricted.

It has been repeatedly shown that SCPs have modalityand task-specific topographies (for recent overviews see $[2,30])$. Also, both the time-course and the location of scalp-recorded negative SCPs showed a close correlation with simultaneously recorded single cell activity in the mammalian cortex [6]. In addition, a good correspondence with measures of regional cerebral blood flow (rCBF) was observed, with the scalp maximum of SCPs being located over those areas where maximal increase in rCBF was observed [19]. Thus, although the spatial resolution of SCPs may be limited to some extent (as it is the case for all surface recordings), one might nevertheless assume that SCPs reveal information about underlying cortical activity which is sufficiently precise to conclude that differences in scalp topographies reflect differences in underlying generator structures. Hence, it can be concluded that the differences between experiments and groups described in this communication also reflect differences in cortical activity. Involvement of parietal cortex in visuo-spatial imagery and mental rotation has been identified in a number of studies using both tomographic and topographic brain mapping techniques [5,7,17,18,28,30,33,35-37]. Richter et al. [28], for instance, used time-resolved functional magnetic resonance imaging (fMRI) to investigate cortical activity during comparison of three-dimensional objects via mental rotation. Since the width of the hemodynamic response in parietal areas was directly related to reaction time and not only to aspects of the task which were constant from trial to trial (such as the visual presentation of the objects or responding to a task), they concluded that parietal cortex is likely involved in the very process of mental rotation. Using fMRI, it was also observed that the amount of activity in superior parietal lobule was positively correlated with the error rate of a task [33]. This finding was interpreted as reflecting the increased demands for information processing evoked by increases in task difficulty. Similarly, it was demonstrated using SCPs [30] that activity of parietal areas is related to the difficulty of a visuo-spatial imagery task, with negative SCP amplitudes of parietal and central leads being higher in trials requiring 2-dimensional mental rotation through larger angles. Therefore, since parietal activity seems to be modulated by task-specific characteristics of visuo-spatial imagery tasks, and since it has been observed in all previous studies as well as in all conditions and in all groups in our own experiments, it seems justified to relate parietal cortex 
activity to 'genuine' task-specific processing. However, there exists some controversy about the involvement of frontal areas in visuo-spatial imagery and mental rotation. Contrary to a positron emission tomography (PET) study specifically designed to assess frontal activity during mental rotation [17], several fMRI studies [5,7] found activity in frontal areas during visuo-spatial imagery, albeit only in some of their subjects. In these studies, frontal activity was assigned to more general (task-unspecific) functional requirements, such as increased directed attention or effort, respectively. Although the cerebral location of the generators of frontal SCPs in our experiments might not be known exactly (candidates being the rostral medial frontal cortex and/or lateral premotor/supplementary motor areas, as suggested by recent findings $[29,36])$, this interpretation seems to be supported by the results of our experiments. Here, subjects showed frontal cortex involvement only when they were sufficiently challenged by task processing. Since poor performers have general difficulties in solving visuo-spatial imagery tasks, this might have been the case irrespective of processing mode for them. Contrary, good performers showed frontal activity only when they were required to respond within a limited time, which might reflect that tasks were sufficiently demanding for these subjects only under the speed condition. Following these arguments, one might speculate that frontal cortex activity was not consistently found in previous investigations $[5,7,17]$ because only some of the subjects of these studies have been sufficiently challenged by the tasks. However, the modulation of cortical activity observed in this study might alternatively be explained by changes in the task-processing strategy [11]. Strategyrelated changes in cortical activity were repeatedly revealed using electrophysiological recordings [21,32]. Hence, whether the increase in frontal cortex activity observed in the present study is related to increased directed attention or effort, to the adoption of a different processing strategy, or even to both, remains to be explored.

If the instruction to answer as quickly and accurately as possible affects cortical activity during solving of a task requiring higher-order cognitive processing has not been investigated systematically before. However, it is this type of cognitive processing which is now increasingly investigated using functional neuroimaging techniques such as PET or fMRI (see, e.g., Fig. 1 in the recent extensive review by Cabeza and Nyberg [4]). The results of the present study clearly demonstrate that additional neurocognitive activities are evoked when subjects are required to solve a task under processing restrictions. These activities are presumably not specifically attributable to the task under investigation, but to rather general processing requirements imposed by speeded task processing. Thus, restricting task processing time might considerably confound the functional interpretation and generalizability of functional neuroimaging experiments. We therefore suggest that this should be taken into account more explicitly in the design and interpretation of future neuroimaging experiments aiming to localize task-specific activity.

\section{Acknowledgements}

We would like to thank Michael and Ulrich Leodolter for their support in data recording and analysis, and Brigitte Röder for her comments on an earlier draft of this paper.

\section{References}

[1] M. Annett, Left, Right, Hand, and Brain: the Right Shift Theory, Erlbaum, London, 1992.

[2] H. Bauer, Slow potential topography, Behavior Res. Meth. Instrum. Comput. 30 (1998) 20-33.

[3] R.J. Boik, A priori tests in repeated measures designs: effects of nonsphericity, Psychometrika 46 (1981) 241-255.

[4] R. Cabeza, L. Nyberg, Imaging cognition II: an empirical review of 275 PET and fMRI studies, J. Cogn. Neurosci. 12 (2000) 1-47.

[5] P.A. Carpenter, M. Just, T.A. Keller, W. Eddy, Graded functional activation in the visuospatial system with the amount of task demand, J. Cogn. Neurosci. 11 (1999) 9-21.

[6] H. Caspers, E.J. Speckmann, A. Lehmenkühler, Electrogenesis of cortical DC potentials, in: H.H. Kornhuber, L. Deecke (Eds.), Motivation, Motor and Sensory Processes of the Brain. Electrical Potentials, Behavior and Clinical Use. Progress in Brain Research, Vol. 54, Elsevier, Amsterdam, pp. 3-16.

[7] M.S. Cohen, S.M. Kosslyn, H.C. Breiter, G.J. DiGirolamo, W.L. Thompson, A.K. Anderson, S.Y. Bookheimer, B.R. Rosen, J.W. Belliveau, Changes in cortical activity during mental rotation: a mapping study using functional MRI, Brain 119 (1996) 89-100.

[8] M. Cohen, M. Kubovy, Mental rotation, mental representation, and flat slopes, Cognit. Psychol. 25 (1993) 351-382.

[9] M.C. Corballis, N.J. Zebrodoff, L.I. Shetzer, B.B. Butler, Decisions about identity and orientation of rotated letters and digits, Memory Cognition 6 (1978) 98-107.

[10] M. Falkenstein, J. Hohnsbein, J. Hoormann, Time pressure effects on late components of the event-related potential (ERP), J. Psychophysiol. 8 (1994) 22-30.

[11] P. Fitts, The information capacity of the human motor system in controlling the amplitude of movement, J. Exp. Psychol. 47 (1954) 381-391.

[12] G. Gittler, Dreidimensionaler Würfeltest (3dw). Theoretische Grundlagen Und Manual, [Three-dimensional Cube Test. Theoretical Foundations and Manual], Beltz Test, Weinheim, 1990.

[13] S.W. Greenhouse, S. Geisser, On methods in the analysis of profile data, Psychometrika 24 (1959) 95-112.

[14] H. Gulliksen, Theory of Mental Tests, 5th Edition, Wiley, New York, 1965.

[15] A.R. Haig, E. Gordon, S. Hook, To scale or not to scale: McCarthy and Wood revisited, Electroenceph. Clin. Neurophysiol. 103 (1997) 323-325.

[16] J. Hohnsbein, M. Falkenstein, J. Hoormann, Effects of attention and time-pressure on P300 subcomponents and implications for mental workload research, Biol. Psychol. 40 (1995) 73-81.

[17] S.M. Kosslyn, G. DiGirolamo, W.L. Thompson, N.M. Alpert, Mental rotation of objects versus hands: neural mechanisms revealed by positron emission tomography, Psychophysiology 35 (1998) 151-161.

[18] C. Lamm, H. Bauer, O. Vitouch, R. Gstättner, Differences in the 
ability to process a visuo-spatial task are reflected in event-related slow cortical potentials of human subjects, Neurosci. Lett. 269 (1999) 137-140.

[19] W. Lang, M. Lang, M. Podreka, M. Steiner, F. Uhl, E. Suess, Ch. Müller, L. Deecke, DC-potential shifts and regional cerebral blood flow reveal frontal cortex involvement in human visuomotor learning, Exp. Brain. Res. 71 (1988) 353-364.

[20] G. McCarthy, Ch.C. Wood, Scalp distribution of event-related potentials: an ambiguity associated with analysis of variance models, Electroenceph. Clin. Neurophysiol. 62 (1985) 203-208.

[21] L.K. McEvoy, M.E. Smith, A. Gevins, Dynamic cortical networks of verbal and spatial working memory: effects of memory load and task practice, Cerebr. Cortex 8 (1998) 563-574.

[22] A. Neubauer, H.H. Freudenthaler, G. Pfurtscheller, Intelligence and spatiotemporal patterns of event related desynchronisation, Intelligence 20 (1995) 249-266.

[23] P. Nunez, Estimation of large scale neocortical source activity with EEG surface Laplacians, Brain Topogr. 2 (1989) 141-154.

[24] P. Nunez, R.B. Silberstein, P.J. Cadusch, R.S. Wijesinghe, A.F. Westdorp, R. Srinivasan, A theoretical and experimental study of high resolution EEG based on surface Laplacians and cortical imaging, Electroenceph. Clin. Neurophysiol. 90 (1994) 40-57.

[25] F. Perrin, J. Pernier, O. Bertrand, J.F. Echallier, Spherical splines for scalp potential and current source density mapping, Electroenceph. Clin. Neurophysiol. 72 (1989) 184-187.

[26] O. Porebski, A psychological and statistical study of speed and power as variables of human ability, Occup. Psychol. 28 (1954) $218-231$.

[27] G. Rasch, Probabilistic Models For Some Intelligence and Attainment Tests, The University of Chicago Press, Chicago, 1980.

[28] W. Richter, K. Ugurbil, A.P. Georgopoulos, S.-G. Kim, Timeresolved fMRI of mental rotation, NeuroReport 8 (1997) 36973702 .
[29] W. Richter, R. Samorjai, R. Summers, M. Jarmasz, R.S. Menon, J.S. Gati, A.P. Georgopoulos, C. Tegeler, K. Ugurbil, S.-G. Kim, Motor area activity during mental rotation studied by time-resolved singletrial fMRI, J. Cogn. Neurosci. 12 (2000) 310-320.

[30] F. Rösler, M. Heil, B. Röder, Slow negative brain potentials as reflections of specific modular resources of cognition, Biol. Psychol. 45 (1997) 109-141.

[31] S.M. Slobounov, K. Fukada, R. Simon, M. Rearick, W. Ray, Neurophysiological and behavioral indices of time pressure effects on visuomotor task performance, Cogn. Brain Res. 9 (2000) 287298.

[32] M.E. Smith, L.K. McEvoy, A. Gevins, Neurophysiological indices of strategy development and skill acquisition, Cogn. Brain Res. 7 (1999) 389-404.

[33] G.A. Tagaris, S.-G. Kim, J.P. Strupp, P. Andersen, K. Ugurbil, A.P. Georgopoulos, Quantitative relations between parietal activation and performance in mental rotation, NeuroReport 7 (1996) 773-776.

[34] G.P.J. VanBreukelen, E.E. Roskam, A rasch model for speed-accuracy trade-off in time limited tests, in: J.-P. Doignon, J.E. Falmagne (Eds.), Mathematical Psychology. Current Developments, Springer, New York, 1991, pp. 251-272.

[35] O. Vitouch, H. Bauer, G. Gittler, M. Leodolter, U. Leodolter, Cortical activity of good and poor spatial test performers during spatial and verbal processing studied with slow potential topography, Int. J. Psychophysiol. 27 (1997) 183-199.

[36] C. Windischberger, C. Lamm, H. Bauer, E. Moser, Whole-cortex fMRI with single-trial analysis of a mental rotation paradigm at 3 Tesla, Neuroimage 11 (2000) S75.

[37] A. Yoshino, M. Inoue, A. Suzuki, A topographic electrophysiologic study of mental rotation, Cogn. Brain Res. 9 (2000) 121-124. 